\title{
Prevalence and Assessment of Severity of Depression Among Ischemic Heart Disease Patients Attending Outpatient Cardiology Department Baghdad Teaching Hospital, Baghdad, Iraq
}

\author{
Shalan Joodah Al-Abbudi*, Faris Hassan Lami and Zainab Abd Wady
}

Department of Psychiatry, College of Medicine Al- Nahrain University, Imamain Kadhimain Medical City, Baghdad, Iraq

\section{Abstract}

Background: Depression and ischemic heart disease present a major comorbidity.

Objective: To determine the prevalence and to estimate the severity of depression, and some of sociodemographic variables among patients with ischemic heart disease.

Methods: A cross-sectional study conducted in Baghdad Teaching Hospital, Baghdad, Iraq. Study included al patients with ischemic heart disease excluding those with unstable medical illness. Socio-demographic variables, history of comorbid illnesses and complications were compiled. Self-Reporting Questionnaire (SRQ-20) to identify mental illnesses; DSM-IV criteria for depression and Hamilton-17 Scale for severity of depression, were used.

Results: A total of 271 patients were approached; $94.1 \%$ responded. About a half was having myocardial infarction (50.6\%), stable angina (31.4\%), and (18\%) unstable angina. $45.1 \%$ had depression; $14.9 \%$ mild, $20 \%$ moderate, $7.06 \%$ sever, and $3.14 \%$ very severe depression. Depressed was significantly associated with age $(P=0.008)$, sex $(P=0.000)$, marital status $(P=0.026)$, occupation $(P=0.000)$, education $(P=0.005)$, income $(P=0.000)$, duration of ischemia $(P=0.001)$, comorbidity with other illnesses $(P=0.000)$, cardiac surgery $(P=0.025)$, and stressful life events $(P=0.000)$.

Conclusion: Depression is high among ischemic heart disease patients, significantly associated with demographic variables. Treating physicians and cardiologists need to be aware of this co-morbidity.

Keywords: Prevalence; Depression; Outpatient; Ischemic heart disease

\section{Introduction}

The prevalence of depression in patients with coronary artery disease is between $19 \%-47 \%$ compared to $4 \%-7 \%$ in the general population [1]. Depression and Coronary Artery Disease (CAD) are both extremely prevalent diseases that compromised quality of life and life expectancy [2]. It has long been recognized that mild forms of depression are found in up to two thirds of patients in hospital after acute myocardial infarction (AMI), with major depression generally being found in about $15 \%$ of CVD patients [3]. There are a number of psychological reactions that can potentially occur after acute medical events. 13 Depressed moods is commonly experienced as a reaction to an acute coronary event, or for that matter to any illness or operation perceived to threaten one's life and well-being [4]. According to World Health Organization's (WHO) estimate, depression and cardiovascular disease will be the two major causes of disability-adjusted life years by the year 2020. According to the Diagnostic and Statistical Manual of Mental Disorders IV (DSM-IV), approximately 25\% of the people with general medical conditions will become depressed during the course of their chronic condition [5]. The relationship between coronary heart disease (CHD) and major depressive disorder (MDD) has been investigated extensively over recent decades, as the prevalence of both conditions has risen around the world. Each condition remains a major contributor to the global burden of disease [6]. Though there are some inconsistencies in the literature, there are several established risk factors for depression in cardiac patients. Most studies have found that younger patients, women, and patients with premorbid histories of depression are more likely to have depression in the context of CVD. Among patients suffering from an acute coronary syndrome (ACS), social isolation and prior ACS, may also increase depression risk [7].
There is a clear consensus that depression is currently underdiagnosed in cardiac patients by cardiologists and primary care physicians alike. There is need for psychological intervention to manage and control the symptoms of depression in cardiovascular diseases in each and every cardiology units [8]. Due to the high prevalence, routine screening for depression in all patients with CHD is recommended when a patient first presents, and at the follow up appointment [3].

\section{Objectives}

This study was conducted with an aim to determine the prevalence and to estimate the severity of depression, and some of sociodemographic variables among patients with ischemic heart disease attending outpatient cardiology department, Baghdad teaching hospital, Baghdad, Iraq.

\section{Patients and Methods}

\section{Design and setting}

This is a cross-sectional study with analytic component. It was

*Corresponding author: Shalan Joodah Al-Abbudi, Department of Psychiatry, College of Medicine Al- Nahrain University, Imamain Kadhimain Medical City, Baghdad, Iraq, Tel: +9647810705221; E-mail: shalanjoodah@gmail.com.

Received: November 05, 2017; Accepted: December 06, 2017; Published: December 16, 2017

Citation: Al-Abbudi SJ, Lami FH, Wady ZA (2018) Prevalence and Assessment of Severity of Depression Among Ischemic Heart Disease Patients Attending Outpatient Cardiology Department Baghdad Teaching Hospital, Baghdad, Iraq. J Psychiatry 21: 438. doi:10.4172/2378-5756.1000438

Copyright: (c) 2018 Al-Abbudi SJ, et al. This is an open-access article distributed under the terms of the Creative Commons Attribution License, which permits unrestricted use, distribution, and reproduction in any medium, provided the original author and source are credited 
conducted in Baghdad Teaching Hospital, Baghdad, Iraq. The data collection was done during the period March $1^{\text {st }} 2011$ to June $30^{\text {th }} 2011$.

\section{Study population and sampling technique}

The study included patients attending the cardiology outpatient clinic at Baghdad teaching hospital during the data collection time. A systematic random sampling technique was applied, where every $3^{\text {rd }}$ patient entering the clinic who accepts to participate in the study and to have the interview was selected.

\section{Inclusion criteria}

All patients with ischemic heart disease of any age and sex who welcomed participation were included.

\section{Exclusion criteria}

Current serious or unstable medical illnesses with severe chest pain or shortness of breath that cannot complete the interview were excluded.

\section{Data collection tools}

Basic socio-demographic variables, diagnosing methods, history of comorbid illnesses and complications were compiled using a questionnaire filled through a direct interview. Mental status of the patient was assessed using the SRQ-20 scale (self- reporting questionnaires) that was developed by the WHO and used in many countries. According to previous studies conducted in Iraq, the cutoff point identified used to categories "potential psychiatric cases" and more generally persons with significant psychological distress was seven [9]. Those with positive results were assessed for the presence of depression using the DSM-IV criteria of depression [10]. Those with "depression" were further assessed for the severity of depression using the Hamilton scale. It contains 17 items to be rated (HRSD-17). Each item on the questionnaire is scored on a 3 or 5 point scale, depending on the item, and the total score is compared to the corresponding descriptor. It is accepted by most clinicians that scores between 0 and 6 do not indicate the presence of depression, scores between 7 and 17 indicate mild depression, scores between 18 and 24 indicate moderate depression, and scores over 24 indicate severe depression. A total HAM-D score of 7 or less after treatment is for most raters a typical indicator of remission [11].

\section{Definition of variables}

The independent variables evaluated to explain depression were socio-demographics (age, gender, marital status, level of education, and occupation), smoking habits, characteristics of the disease, and comorbid condition or disease.

\section{Statistical analysis}

Statistical package of social sciences (SPSS) version 15 was used for data entry and analysis. Categorical variables were tested using chi square test. $\mathrm{P}<0.05$ was considered statistically significant.

\section{Ethical issue}

Official approvals were granted from the officials in the study setting. Informed consent was obtained from each participant to be included in this study. Names were kept anonymous and interviews were conducted with full privacy.

\section{Limitations of the study}

Random sampling was not applied to choose cardiology clinic.
The study was conducted in one cardiology clinic. Since this study is a cross-sectional study. Temporal relationship between depression and ischemic heart disease cannot be inferred. Similarly, selection bias cannot be excluded as the study was conducted in a specialized center.

\section{Results}

A total of 271 ischemic heart disease patients were approached; 255 accepted to participate, giving a response rate of (94.1\%). About $70.2 \%$ of the sample was male; $79.6 \%$ were married; $39.6 \%$ were still working; and about $75.3 \%$ had poor education. About $90 \%$ were live in urban than rural areas. Myocardial infarction 50.6\%, stable angina $31.4 \%$, and $18 \%$ were have unstable angina. Distribution by socio-demographic and ischemic heart disease participants characteristics are shown in Table 1.

The mean age of patients was $(58.945 \pm 11.026)$ years. The family size mean was $(8.76 \pm 5.2)$, crowding index mean was $(3.07 \pm 4.21)$, and the average $( \pm \mathrm{SD})$ monthly family income was $(533.3 \pm 56.9)$ ID (Table 2).

The methods used in the diagnosis of ischemic heart disease were depend mostly on the sign and symptoms of chest pain and ECG changes $(98.9 \%)$, admission to CCU (78.8\%), other investigations like treadmill test (24.7\%) and Echocardiograph study (6.6\%) (Table 3).

The diagnosis of ischemic heart disease was associated with high comorbidity of other illnesses $(72.5 \%)$. The majority show comorbidity with hypertension (50.9\%), Diabetes Mellitus (33.7\%), asthmatic bronchitis (1.56\%), heart failure (9.4\%), cerebrovascular accidents (3.1\%), and duodenal ulceration (1.56\%) (Table 4).

Nearly one third of the participants were smoker (33.3\%), range 3-100 cigarettes per day, the most frequent is $10-40$ cigarettes per day $(26.3 \%)$. The sample shows $3.2 \%$ were alcohol drinker of regular drinking habits (Table 5).

More than half of the participants exposed to one of the major life events (54.5); $21.6 \%$ exposed to death of close relative; $7.9 \%$ death of spouse, $20.4 \%$ money loss, $18.9 \%$ serious illness or accidents and $0.8 \%$ exposed to job loss (Table 6).

All participants were on treatment; antiplatelet (95.3\%), nitrate (93.4\%), lipid lowering agents (67.9\%), B-blocker (62.8\%), ACE inhibitors (45.6\%), diuretics (27.9\%), calcium channel blocker (17.3\%), angiotensin (7.6\%), and anticoagulants (3.2\%) (Table 7).

SRQ-20 positive responses were (145) (56.86\%); stable angina (52) (63.4\%), myocardial infarction (96) (53.4\%), unstable angina (24) (52.1\%) (Table 8).

Participants show 115 (45.1\%) were met DSM-IV criteria of depressed; 51 (44.3\%) of them were myocardial infarction, 42 (36.5\%) stable angina, and 22 (19.1\%) were unstable angina (Table 9).

The severity of depression was investigated by applying the Hamilton depression scale (HAMD-17) among positive DSM-IV criteria of depression found that; 38 (14.9\%) mild depression, 51 (20\%) moderate depression, 18 (7.06\%) severe depression, and 8 (3.14\%) very severe depression. Moderate and severe depression was more among myocardial infarction while very severe depression was among stable angina (Table 10)

Depressed ischemic heart disease participants were more among 5160 years age group (32.2\%), male (53\%) compared with female (47\%). Depression found more within married (74\%), widowed (24.3\%), and 
Citation: Al-Abbudi SJ, Lami FH, Wady ZA (2018) Prevalence and Assessment of Severity of Depression Among Ischemic Heart Disease Patients Attending Outpatient Cardiology Department Baghdad Teaching Hospital, Baghdad, Iraq. J Psychiatry 21: 438. doi:10.4172/23785756.1000438

Page 3 of 10

\begin{tabular}{|c|c|c|c|c|c|c|c|c|c|c|}
\hline & & \multicolumn{6}{|c|}{ Classification of Ischemia } & \multirow{3}{*}{ Total } & \multirow{3}{*}{$\%$} & \multirow{3}{*}{$P$ value } \\
\hline & & \multicolumn{2}{|c|}{ stable angina } & \multicolumn{2}{|c|}{ unstable angina } & \multicolumn{2}{|c|}{ myocardial infarction } & & & \\
\hline & & No. & $\%$ & No. & $\%$ & No. & $\%$ & & & \\
\hline \multirow{7}{*}{ Age Groups } & $\leq 30$ Years & 0 & 0 & 0 & 0 & 4 & 3.1 & 4 & 1.6 & \multirow{7}{*}{0.083} \\
\hline & $31-40$ Years & 2 & 2.5 & 0 & 0 & 6 & 4.7 & 8 & 3.1 & \\
\hline & $41-50$ Years & 12 & 15 & 8 & 17.4 & 28 & 21.8 & 48 & 18.8 & \\
\hline & $51-60$ Years & 20 & 25 & 20 & 43.5 & 35 & 27.1 & 75 & 29.4 & \\
\hline & $61-70$ Years & 28 & 35 & 16 & 34.8 & 40 & 31 & 84 & 33 & \\
\hline & 71 - 80 Years & 16 & 20 & 2 & 4.3 & 14 & 10.8 & 32 & 12.5 & \\
\hline & $81+$ Years & 2 & 2.5 & 0 & 0 & 2 & 1.5 & 4 & 1.6 & \\
\hline \multirow{2}{*}{ Sex } & Male & 42 & 52.5 & 34 & 73.9 & 103 & 79.9 & 179 & 70.2 & \multirow{2}{*}{0.000} \\
\hline & Female & 38 & 47.5 & 12 & 26.1 & 26 & 20.1 & 76 & 29.8 & \\
\hline \multirow{3}{*}{ Marital Status } & Single & 6 & 7.5 & 0 & 0 & 0 & 0 & 6 & 2.4 & \multirow{3}{*}{0.000} \\
\hline & Married & 52 & 65 & 38 & 82.6 & 113 & 87.6 & 203 & 79.6 & \\
\hline & Widowed & 22 & 27.5 & 8 & 17.4 & 16 & 12.4 & 46 & 18 & \\
\hline \multirow{6}{*}{ Occupation } & unemployed & 2 & 2.5 & 6 & 13 & 6 & 4.6 & 14 & 5.5 & \multirow{6}{*}{0.000} \\
\hline & Gov. Work & 4 & 5 & 6 & 13 & 12 & 9.3 & 22 & 8.6 & \\
\hline & Private Work & 12 & 15 & 16 & 34.8 & 41 & 31.8 & 69 & 27.1 & \\
\hline & Military & 2 & 2.5 & 0 & 0 & 8 & 6.2 & 10 & 3.9 & \\
\hline & Retired & 24 & 30 & 8 & 17.4 & 40 & 31 & 72 & 28.2 & \\
\hline & Housewife & 36 & 45 & 10 & 21.8 & 22 & 17.1 & 68 & 26.7 & \\
\hline \multirow{4}{*}{ Education } & Illiterate & 34 & 42.5 & 12 & 26.1 & 30 & 23.2 & 76 & 29.8 & \multirow{4}{*}{0.031} \\
\hline & Primary & 26 & 32.5 & 26 & 56.5 & 64 & 49.6 & 116 & 45.5 & \\
\hline & Secondary & 10 & 12.5 & 6 & 13 & 22 & 17.1 & 38 & 14.9 & \\
\hline & university & 10 & 12.5 & 2 & 4.4 & 13 & 10.1 & 25 & 9.8 & \\
\hline Total & & 80 & $100 \%$ & 46 & $100 \%$ & 129 & $100 \%$ & 255 & $100 \%$ & \\
\hline
\end{tabular}

Table 1: Show the socio-demographic characteristic features of the participants according to the type of ischemia.

\begin{tabular}{|c|c|c|c|}
\hline Variables & N & Mean & Median \\
\hline Age & 255 & 58.94 & 60.00 \\
\hline Family size & 255 & 8,76 & 8.00 \\
\hline Crowding Index & 255 & 3.07 & 5.20 \\
\hline Monthly family income (ID) & 255 & 533302 & 3.21 \\
\hline
\end{tabular}

Table 2: Mean, median and standard deviation of some sociodemographic features of the sample.

\begin{tabular}{|c|c|c|c|c|c|c|}
\hline \multirow{2}{*}{\multicolumn{2}{|c|}{ Way of Diagnosis }} & \multicolumn{3}{|c|}{ Classification of Ischemia } & \multirow{3}{*}{$\begin{array}{c}\text { Total } \\
3 \\
\end{array}$} & \multirow{4}{*}{$\begin{array}{c}\text { P value } \\
0.227\end{array}$} \\
\hline & & \multirow{2}{*}{$\begin{array}{c}\text { stable angina } \\
0\end{array}$} & \multirow{2}{*}{$\begin{array}{c}\text { unstable angina } \\
0\end{array}$} & \multirow{2}{*}{$\begin{array}{c}\text { myocardial infarction } \\
3\end{array}$} & & \\
\hline chest pain & Negative & & & & & \\
\hline & Positive & 80 & 46 & 126 & 252 & \\
\hline \multirow[t]{2}{*}{ ECG changes } & Negative & 0 & 0 & 3 & 3 & \multirow{2}{*}{0.227} \\
\hline & Positive & 80 & 46 & 126 & 252 & \\
\hline \multirow[t]{2}{*}{ CCU admission } & Negative & 34 & 4 & 16 & 54 & \multirow{2}{*}{0.000} \\
\hline & Positive & 46 & 42 & 113 & 201 & \\
\hline \multirow[t]{2}{*}{ Treadmill Test } & Negative & 60 & 40 & 92 & 192 & \multirow{2}{*}{0.107} \\
\hline & Positive & 20 & 6 & 37 & 63 & \\
\hline \multirow[t]{2}{*}{ Catheterization } & Negative & 48 & 26 & 67 & 141 & \multirow{2}{*}{0.514} \\
\hline & Positive & 32 & 20 & 62 & 114 & \\
\hline \multirow[t]{2}{*}{ Echo Study } & Negative & 68 & 44 & 126 & 238 & \multirow{2}{*}{0.001} \\
\hline & Positive & 12 & 2 & 3 & 17 & \\
\hline \multicolumn{2}{|c|}{ Total } & 80 & 46 & 129 & 255 & \\
\hline
\end{tabular}

Table 3: The way of diagnosis of ischemic heart disease among the study participants.

single (1.7\%). Regarding the occupation founded that house wife form $41.7 \%$ of the depressed participants, while retired (24.3\%), private work (21.7\%), and unemployed (7\%). Depression founded more among low educated participants; illiterate (43.5\%) and primary school $(41.7 \%)$ (Table 11)

Of the 115 (45.1\%) depressed participants; myocardial infarction
(44.3\%), stable angina (36.5\%), and unstable angina (19.1\%). the correlation between the degree of depression and the type of ischemia was of highly statistical significant (Tables 12 and 13).

Depressed participants were of mean age \pm SD $=58.945 \pm 11.02$ years, the monthly income by Iraqi dinar $=533.301 \pm 56.951$. The living circumstances at the home atmosphere; the number of persons at one 
Citation: Al-Abbudi SJ, Lami FH, Wady ZA (2018) Prevalence and Assessment of Severity of Depression Among Ischemic Heart Disease Patients Attending Outpatient Cardiology Department Baghdad Teaching Hospital, Baghdad, Iraq. J Psychiatry 21: 438. doi:10.4172/23785756.1000438

Page 4 of 10

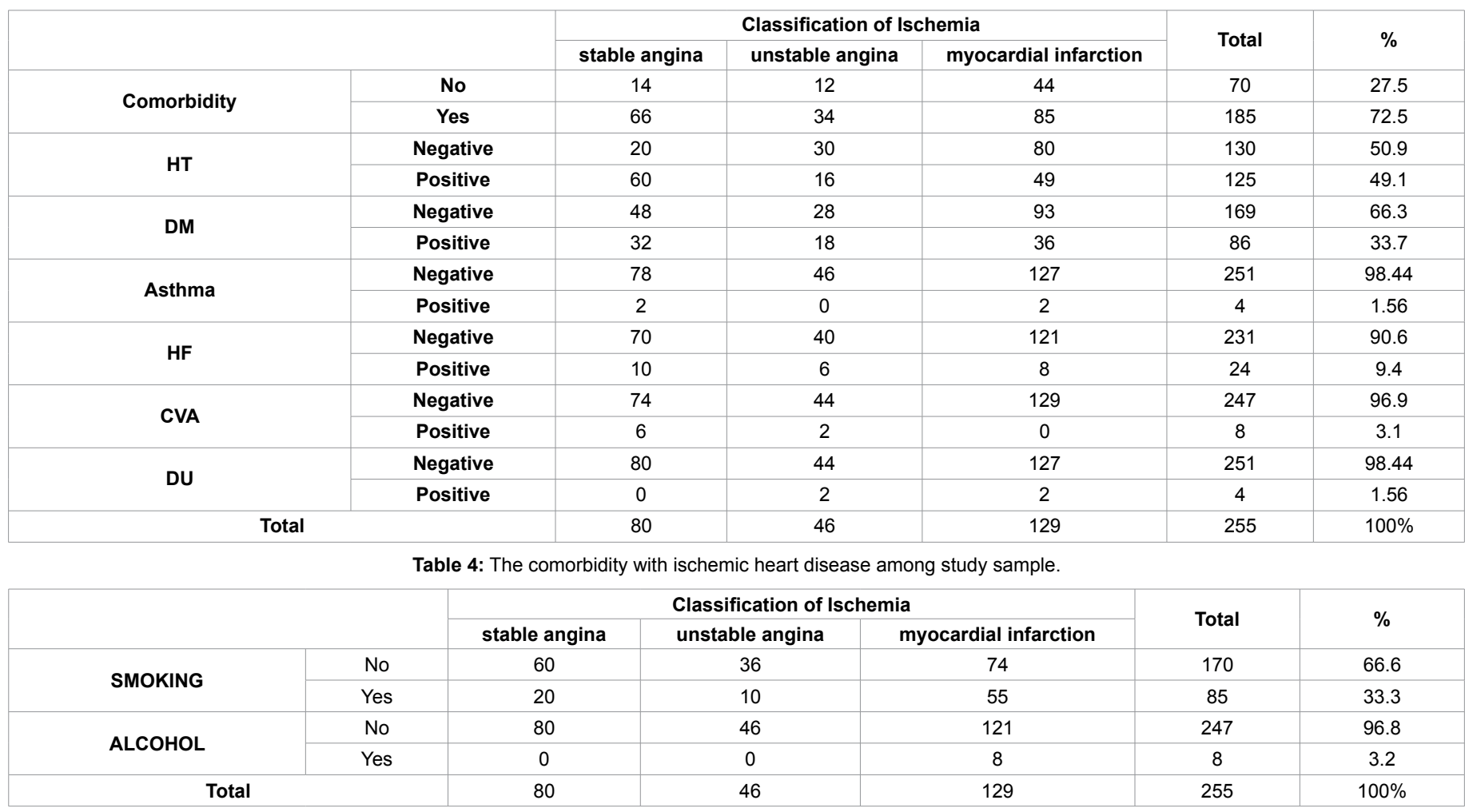

Table 5: Smoking and alcohol drinking with ischemic heart disease among participants of the study.

\begin{tabular}{|c|c|c|c|c|c|c|}
\hline & & \multicolumn{3}{|c|}{ Classification of Ischemia } & \multirow{2}{*}{ Total } & \multirow{2}{*}{$\%$} \\
\hline & & stable angina & unstable angina & myocardial infarction & & \\
\hline \multirow{2}{*}{ Life Events } & Negative & 30 & 42 & 62 & 116 & 45.5 \\
\hline & Positive & 50 & 22 & 67 & 139 & 54.5 \\
\hline \multirow{2}{*}{ Death of close relative } & Negative & 58 & 36 & 106 & 200 & 78.4 \\
\hline & Positive & 22 & 10 & 23 & 55 & 21.6 \\
\hline \multirow{2}{*}{ Death of Spouse } & Negative & 70 & 44 & 121 & 235 & 92.1 \\
\hline & Positive & 10 & 2 & 8 & 20 & 7.9 \\
\hline \multirow[t]{2}{*}{ Money Loss } & Negative & 62 & 38 & 103 & 203 & 79.6 \\
\hline & Positive & 18 & 8 & 26 & 52 & 20.4 \\
\hline \multirow{2}{*}{ Job Loss } & Negative & 78 & 46 & 129 & 253 & 99.2 \\
\hline & Positive & 2 & 0 & 0 & 2 & 0.8 \\
\hline \multirow{2}{*}{ Home Change } & Negative & 74 & 46 & 125 & 245 & 96.07 \\
\hline & Positive & 6 & 0 & 4 & 10 & 3.93 \\
\hline \multirow{2}{*}{ Serious illness or accident } & Negative & 64 & 38 & 105 & 207 & 81.1 \\
\hline & Positive & 16 & 8 & 24 & 48 & 18.9 \\
\hline \multicolumn{2}{|l|}{ Total } & 80 & 46 & 129 & 255 & $100 \%$ \\
\hline
\end{tabular}

Table 6: Stressful life events associated with ischemic heart disease among study sample.

\begin{tabular}{|c|c|c|c|c|c|c|}
\hline & & \multicolumn{3}{|c|}{ Classification of Ischemia } & \multirow{2}{*}{ Total } & \multirow{2}{*}{$\%$} \\
\hline & & stable angina & unstable angina & myocardial infarction & & \\
\hline \multirow[t]{2}{*}{ B Blocker } & No & 30 & 20 & 45 & 95 & 37.2 \\
\hline & Yes & 50 & 26 & 84 & 160 & 62.8 \\
\hline \multirow{2}{*}{ Lipid Lowering Agents } & No & 32 & 16 & 34 & 82 & 32.1 \\
\hline & Yes & 48 & 30 & 95 & 173 & 67.9 \\
\hline \multirow{2}{*}{ Nitrate } & No & 8 & 2 & 7 & 17 & 6.6 \\
\hline & Yes & 72 & 44 & 122 & 238 & 93.4 \\
\hline \multirow{2}{*}{ ACE inhibitor } & No & 40 & 26 & 73 & 139 & 54.5 \\
\hline & Yes & 40 & 20 & 56 & 116 & 45.6 \\
\hline \multirow{2}{*}{ Angiotensin } & No & 70 & 46 & 120 & 236 & 92.5 \\
\hline & Yes & 10 & 0 & 9 & 19 & 7.6 \\
\hline \multirow{2}{*}{ Antiplatelate } & No & 2 & 4 & 6 & 12 & 4.7 \\
\hline & Yes & 78 & 42 & 123 & 243 & 95.3 \\
\hline
\end{tabular}


Citation: Al-Abbudi SJ, Lami FH, Wady ZA (2018) Prevalence and Assessment of Severity of Depression Among Ischemic Heart Disease Patients Attending Outpatient Cardiology Department Baghdad Teaching Hospital, Baghdad, Iraq. J Psychiatry 21: 438. doi:10.4172/23785756.1000438

Page 5 of 10

\begin{tabular}{|c|c|c|c|c|c|c|}
\hline \multirow{2}{*}{ Calcium Channel Blocker } & No & 68 & 42 & 101 & 211 & 82.7 \\
\hline & Yes & 12 & 4 & 28 & 44 & 17.3 \\
\hline \multirow{2}{*}{ Diuretics } & No & 52 & 28 & 104 & 184 & 72.1 \\
\hline & Yes & 28 & 18 & 25 & 71 & 27.9 \\
\hline \multirow{2}{*}{ Anticoagulant } & No & 76 & 46 & 125 & 247 & 96.8 \\
\hline & Yes & 4 & 0 & 4 & 8 & 3.2 \\
\hline \multicolumn{2}{|l|}{ Total } & 80 & 46 & 129 & 255 & $100 \%$ \\
\hline
\end{tabular}

Table 7: Type of medication taken by ischemic heart disease participants of the study.

\begin{tabular}{|c|c|c|c|c|c|c|c|c|c|}
\hline \multirow{4}{*}{ SRQ Responses } & \multirow[b]{3}{*}{ Negative } & \multicolumn{6}{|c|}{ Classification of Ischemia } & \multirow{2}{*}{\multicolumn{2}{|c|}{ Total }} \\
\hline & & \multicolumn{2}{|c|}{ stable angina } & \multicolumn{2}{|c|}{ unstable angina } & \multicolumn{2}{|c|}{ myocardial infarction } & & \\
\hline & & 28 & $11 \%$ & 22 & $8.6 \%$ & 60 & $23.5 \%$ & 110 & $43.1 \%$ \\
\hline & Positive & 52 & $20.3 \%$ & 24 & $9.4 \%$ & 69 & $27 . \%$ & 145 & $56.9 \%$ \\
\hline \multicolumn{2}{|c|}{ Total } & 80 & $31.3 \%$ & 46 & $18 \%$ & 129 & $50.5 \%$ & 255 & $100 \%$ \\
\hline
\end{tabular}

Table 8: SRQ-20 positive and negative responses among ischemic heart disease participants of the study.

\begin{tabular}{|c|c|c|c|c|c|c|c|c|c|}
\hline \multirow{4}{*}{ Depression } & \multirow[b]{3}{*}{ No } & \multicolumn{6}{|c|}{ Classification of Ischemia } & \multirow{3}{*}{$\begin{array}{c}\text { Total } \\
140 \\
\end{array}$} & \multirow{3}{*}{$\begin{array}{c}\% \\
54.9 \% \\
\end{array}$} \\
\hline & & \multicolumn{2}{|c|}{ stable angina } & \multicolumn{2}{|c|}{ unstable angina } & \multicolumn{2}{|c|}{ myocardial infarction } & & \\
\hline & & 38 & $14.9 \%$ & 24 & $9.4 \%$ & 78 & $30.5 \%$ & & \\
\hline & Yes & 42 & $16.4 \%$ & 22 & $8.6 \%$ & 51 & $20 \%$ & 115 & $45.1 \%$ \\
\hline \multicolumn{2}{|c|}{ Total } & 80 & $31.3 \%$ & 46 & $18 \%$ & 129 & $50.5 \%$ & 255 & $100 \%$ \\
\hline
\end{tabular}

Table 9: Depression among ischemic heart disease participants of the study.

\begin{tabular}{|c|c|c|c|c|c|c|c|c|c|c|}
\hline & & \multicolumn{6}{|c|}{ Classification of Ischemia } & \multirow{2}{*}{ Total } & \multirow{2}{*}{$\%$} & \multirow{2}{*}{$P$ value } \\
\hline & & \multicolumn{2}{|c|}{ stable angina } & \multicolumn{2}{|c|}{ unstable angina } & \multicolumn{2}{|c|}{ myocardial infarction } & & & \\
\hline \multirow{5}{*}{ HAMD Degree } & Not done & 38 & $14.9 \%$ & 24 & $9.4 \%$ & 78 & $30.5 \%$ & 140 & 54.9 & \multirow{5}{*}{0.003} \\
\hline & Mild depression & 16 & $6.2 \%$ & 6 & $2.3 \%$ & 16 & $6.2 \%$ & 38 & 14.9 & \\
\hline & Moderate depression & 14 & $5.4 \%$ & 12 & $4.7 \%$ & 25 & $9.8 \%$ & 51 & 20 & \\
\hline & Severe depression & 4 & $1.5 \%$ & 4 & $1.5 \%$ & 10 & $3.9 \%$ & 18 & 7.06 & \\
\hline & $\begin{array}{l}\text { Very severe } \\
\text { depression }\end{array}$ & 8 & $3.1 \%$ & 0 & $0 \%$ & 0 & $0 \%$ & 8 & 3.14 & \\
\hline \multicolumn{2}{|c|}{ Total } & 80 & $31.3 \%$ & 46 & $18.03 \%$ & 129 & $50.5 \%$ & 255 & $100 \%$ & \\
\hline
\end{tabular}

Table 10: The severity of depression among Ischemic heart disease patients obtained from HAMD-17 of the study sample.

\begin{tabular}{|c|c|c|c|c|c|c|c|c|}
\hline & & \multicolumn{4}{|c|}{ Degree of Depression } & \multirow[b]{2}{*}{ Total } & \multirow[b]{2}{*}{$\%$} & \multirow[b]{2}{*}{$P$ value } \\
\hline & & Mild depression & Moderate depression & $\begin{array}{c}\text { Severe } \\
\text { depression }\end{array}$ & $\begin{array}{l}\text { Very severe } \\
\text { depression }\end{array}$ & & & \\
\hline \multirow{7}{*}{ Age Groups } & 26 - 30 Years & 2 & 0 & 0 & 0 & 2 & 1.7 & \multirow{7}{*}{0.003} \\
\hline & $31-40$ Years & 0 & 0 & 2 & 0 & 2 & 1.7 & \\
\hline & 41 - 50 Years & 8 & 8 & 4 & 6 & 26 & 22.6 & \\
\hline & $51-60$ Years & 10 & 21 & 6 & 0 & 37 & 32.2 & \\
\hline & 61 - 70 Years & 10 & 16 & 4 & 0 & 30 & 26 & \\
\hline & 71 - 80 Years & 8 & 4 & 2 & 2 & 16 & 14 & \\
\hline & $81+$ Years & 0 & 2 & 0 & 0 & 2 & 1.7 & \\
\hline \multirow[t]{2}{*}{ Sex } & Male & 24 & 27 & 10 & 0 & 61 & 53 & \multirow{2}{*}{0.014} \\
\hline & Female & 14 & 24 & 8 & 8 & 54 & 47 & \\
\hline \multirow{3}{*}{ Marital Status } & Single & 0 & 2 & 0 & 0 & 2 & 1.7 & \multirow{3}{*}{0.107} \\
\hline & Married & 32 & 33 & 16 & 4 & 85 & 74 & \\
\hline & Widowed & 6 & 16 & 2 & 4 & 28 & 24.3 & \\
\hline \multirow{6}{*}{ Occupation } & Unemployed & 6 & 0 & 2 & 0 & 8 & 7 & \multirow{6}{*}{0.001} \\
\hline & Gov. work & 0 & 4 & 0 & 0 & 4 & 3.5 & \\
\hline & Private Work & 14 & 7 & 2 & 2 & 25 & 21.7 & \\
\hline & Military & 0 & 0 & 2 & 0 & 2 & 1.7 & \\
\hline & Retired & 6 & 16 & 6 & 0 & 28 & 24.3 & \\
\hline & Housewife & 12 & 24 & 6 & 6 & 48 & 41.7 & \\
\hline \multirow{4}{*}{ Education } & Illiterate & 16 & 24 & 6 & 4 & 50 & 43.5 & \multirow{4}{*}{0.432} \\
\hline & Primary & 14 & 20 & 10 & 4 & 48 & 41.7 & \\
\hline & Secondary & 6 & 2 & 2 & 0 & 10 & 8.7 & \\
\hline & University & 2 & 5 & 0 & 0 & 7 & 6.1 & \\
\hline \multicolumn{2}{|c|}{ Total } & 38 & 51 & 18 & 8 & 115 & $100 \%$ & \\
\hline
\end{tabular}

Table 11: Show the socio-demographic features according to the degree of depression which resulted from the HAMD-17. 
Citation: Al-Abbudi SJ, Lami FH, Wady ZA (2018) Prevalence and Assessment of Severity of Depression Among Ischemic Heart Disease Patients Attending Outpatient Cardiology Department Baghdad Teaching Hospital, Baghdad, Iraq. J Psychiatry 21: 438. doi:10.4172/23785756.1000438

Page 6 of 10

\begin{tabular}{|c|c|c|c|c|c|}
\hline & & \multicolumn{2}{|c|}{ Depression } & \multirow{2}{*}{ Total } & \multirow{2}{*}{$P$ value } \\
\hline & & no & Yes & & \\
\hline \multirow{7}{*}{ Age Groups } & 26 - 30 Years & 2 & 2 & 4 & \multirow{7}{*}{0.338} \\
\hline & 31 - 40 Years & 6 & 2 & 8 & \\
\hline & 41 - 50 Years & 22 & 26 & 48 & \\
\hline & 51 - 60 Years & 38 & 37 & 75 & \\
\hline & 61 - 70 Years & 54 & 30 & 84 & \\
\hline & 71 - 80 Years & 16 & 16 & 32 & \\
\hline & $81+$ Years & 2 & 2 & 4 & \\
\hline \multirow{2}{*}{ Sex } & Male & 118 & 61 & 179 & \multirow{2}{*}{0.000} \\
\hline & Female & 22 & 54 & 76 & \\
\hline \multirow{3}{*}{ Marital Status } & Single & 4 & 2 & 6 & \multirow{3}{*}{0.05} \\
\hline & Married & 118 & 85 & 203 & \\
\hline & Widowed & 18 & 28 & 46 & \\
\hline \multirow{6}{*}{ Occupation } & Unemployed & 6 & 8 & 14 & \multirow{6}{*}{0.000} \\
\hline & Govt. work & 18 & 4 & 22 & \\
\hline & Private work & 44 & 25 & 69 & \\
\hline & Military & 8 & 2 & 10 & \\
\hline & Retired & 44 & 28 & 72 & \\
\hline & Housewife & 20 & 48 & 68 & \\
\hline \multirow{4}{*}{ Education } & Illiterate & 26 & 50 & 76 & \multirow{4}{*}{0.000} \\
\hline & Primary & 68 & 48 & 116 & \\
\hline & Secondary & 28 & 10 & 38 & \\
\hline & University & 18 & 7 & 25 & \\
\hline \multicolumn{2}{|c|}{ Total } & 140 & 115 & 255 & \\
\hline
\end{tabular}

Table 12: Sociodemographic features of depressed ischemic heart disease participants of the study.

\begin{tabular}{|c|c|c|c|c|c|c|c|c|}
\hline & & \multicolumn{4}{|c|}{ Degree of Depression } & \multirow[b]{2}{*}{ Total } & \multirow[b]{2}{*}{$\%$} & \multirow[b]{2}{*}{$P$ value } \\
\hline & & mild depression & $\begin{array}{c}\text { moderate } \\
\text { depression }\end{array}$ & $\begin{array}{c}\text { severe } \\
\text { depression }\end{array}$ & $\begin{array}{l}\text { very severe } \\
\text { depression }\end{array}$ & & & \\
\hline \multirow{3}{*}{$\begin{array}{c}\text { Classification of } \\
\text { Ischemia }\end{array}$} & stable angina & 16 & 14 & 4 & 8 & 42 & 36.5 & \multirow{4}{*}{0.006} \\
\hline & unstable angina & 6 & 12 & 4 & 0 & 22 & 19.1 & \\
\hline & myocardial infarction & 16 & 25 & 10 & 0 & 51 & 44.3 & \\
\hline \multicolumn{2}{|c|}{ Total } & 38 & 51 & 18 & 8 & 115 & $100 \%$ & \\
\hline
\end{tabular}

Table 13: The correlation between the degree of depression and the type of ischemic heart disease.

home was $8.76 \pm 5.2$, the number of rooms at one home was $2.85 \pm 1.23$ The duration of ischemic heart disease among depressed participants was $53.38 \pm 68.746$ months. About $33.3 \%$ of the sample was smokers and $32.3 \%$ of depressed participants were smokers with number of cigarette per day $11.435 \pm 19.754$ cigarettes (Tables 14 and 15).

Depressed ischemic heart disease participants show highly significant comorbidity with other diseases. Table 16 show the comorbidity and the statistical significant of the findings.

Depressed ischemic heart disease participants show highly significant association with stressful life events $(\mathrm{P}=0.000)$. About $54.5 \%$ of the whole participants and about $80 \%$ of depressed participants have at least one major life event (Table 17).

Depressed participants of ischemic heart disease patients were of medication either for the ischemia or for comorbidity of other illnesses. Table 18 shows some of the medication that taken by the participants currently and the statistical significance with depression.

More than $90 \%$ of ischemic heart disease patients were live in urban areas than rural areas. All the participants were of negative family history of depression. $2.4 \%$ of the participants were underwent cardiac surgery and no one of them was depressed $(\mathrm{P}=0.025)$ (Table 19).

\section{Discussion}

The study shows response rate of $94.1 \%$. It was similar rates of participation with Salman 2009 (93.6\%) [12] and Iraqi family health survey (IFHS) (95\%) [13]. It was high rate since most of Iraqi people like sharing talk about their conditions where they feel someone take care of them. The other $5.9 \%$ have an excuse not to participate; they feel shame, just in hurry, or have no time.

Participants show (56.86\%) positive responses to SRQ-20. It is higher than many Iraqi studies; Salman 2009 (36.81\%) [12], Muffed et al. (one third) [9], Iraqi mental health survey IMHS (7.14\%) [14], Iraqi family health survey (IFHS) (35.5\%) [13]. High SRQ-20 positive responses may be explained that, Iraqis have witnessed many internal and external conflicts during the past three decades, including three wars and 12 years of sanctions. Since 2003, violence has been increasing throughout the country. These circumstances have affected the Iraqi population in every domain of life with varying degrees of repercussion. This population can be considered as having significant psychological distress and potential psychiatric cases.

Current study founded Depression was $45.1 \%$ of participants with ischemic heart disease. It is higher than; Fraz K et al. 27\% [5], Liang JJ et al. 33\% [14], Raj HSS and Sajimon PP (36.68\%) [8] Iraqi mental health survey (IMHS) 2006/7 [9] (severe depression 3.50\%, moderate depression $2.83 \%$ and mild depression $1.15 \%$ ), Najeb GT [15] $38.67 \%$, and Lawson R et al. [16] 38\%. WHO educational program on depression, founded depression is $33 \%$ among patients with ischemic heart [17]. Leegte IL et al. [18] founded $41 \%$ percent of Netherlands sample had depression. Whooley MA (20\%) [19]. Kemp DE et al. [20] 
Citation: Al-Abbudi SJ, Lami FH, Wady ZA (2018) Prevalence and Assessment of Severity of Depression Among Ischemic Heart Disease Patients Attending Outpatient Cardiology Department Baghdad Teaching Hospital, Baghdad, Iraq. J Psychiatry 21: 438. doi:10.4172/23785756.1000438

Page 7 of 10

\begin{tabular}{|c|c|c|c|c|c|c|}
\hline & \multirow{2}{*}{ Mean } & \multirow{2}{*}{ \pm SD } & \multicolumn{2}{|c|}{ Depression } & \multirow{2}{*}{ Total } & \multirow{2}{*}{$P$ value } \\
\hline & & & NO & YES & & \\
\hline Age & 58.945 & 11.02 & 140 & 115 & 255 & 0.007 \\
\hline Income ID & 533.301 & 56.951 & 140 & 115 & 255 & 0.000 \\
\hline No. of Persons & 8.76 & 5.2 & 140 & 115 & 255 & 0.000 \\
\hline No. of Rooms & 2.85 & 1.23 & 140 & 115 & 255 & 0.020 \\
\hline Duration of illness in Months & 53.38 & 68.746 & 140 & 115 & 255 & 0.001 \\
\hline No. of Cigarette per day & 11.435 & 19.754 & 140 & 115 & 255 & 0.05 \\
\hline
\end{tabular}

Table 14: Some sociodemographic variables of depressed participants with their statistical significant.

\begin{tabular}{|c|c|c|c|c|}
\hline & & \multicolumn{2}{|c|}{ Depression } & \multirow[t]{2}{*}{ Tota } \\
\hline & & NO & YES & \\
\hline \multirow{2}{*}{ SMOKING } & No & 92 & 78 & 170 \\
\hline & Yes & 48 & 37 & 85 \\
\hline \multirow{2}{*}{ ALCOHOL } & No & 134 & 113 & 247 \\
\hline & Yes & 6 & 2 & 8 \\
\hline \multicolumn{2}{|c|}{ Total } & 140 & 115 & 255 \\
\hline
\end{tabular}

Table 15: Sociodemographic variables of depressed participants with their statistical significant.

\begin{tabular}{|c|c|c|c|c|c|}
\hline \multirow{4}{*}{ Co Morbidity } & & \multicolumn{2}{|c|}{ Depression } & \multirow[t]{2}{*}{ Total } & \multirow{2}{*}{$P$ value } \\
\hline & & NO & YES & & \\
\hline & NO & 54 & 16 & 70 & ח \\
\hline & YES & 86 & 99 & 185 & 0.000 \\
\hline \multirow{2}{*}{ HT } & Negative & 86 & 44 & 130 & \multirow{2}{*}{0.000} \\
\hline & Positive & 54 & 71 & 125 & \\
\hline \multirow{2}{*}{ DM } & Negative & 104 & 65 & 169 & \multirow{2}{*}{0.003} \\
\hline & Positive & 36 & 50 & 86 & \\
\hline \multirow{2}{*}{ Asthma } & Negative & 138 & 113 & 251 & \multirow{2}{*}{0.843} \\
\hline & Positive & 2 & 2 & 4 & \\
\hline \multirow{2}{*}{ HF } & Negative & 132 & 99 & 231 & \multirow{2}{*}{0.026} \\
\hline & Positive & 8 & 16 & 24 & \\
\hline \multirow{2}{*}{ CVA } & Negative & 134 & 113 & 247 & \multirow{2}{*}{0.246} \\
\hline & Positive & 6 & 2 & 8 & \\
\hline \multirow{2}{*}{ DU } & Negative & 138 & 113 & 251 & \multirow{2}{*}{0.843} \\
\hline & Positive & 2 & 2 & 4 & \\
\hline \multicolumn{2}{|c|}{ Total } & 140 & 115 & 255 & \\
\hline
\end{tabular}

Table 16: Comorbidity and statistical significant among depressed ischemic heart disease participants.

\begin{tabular}{|c|c|c|c|c|c|}
\hline & & \multicolumn{2}{|c|}{ Depression } & \multirow[t]{2}{*}{ Total } & \multirow{2}{*}{$P$ value } \\
\hline & & NO & YES & & \\
\hline \multirow{2}{*}{ Life Events } & No & 92 & 24 & 116 & \multirow{2}{*}{0.000} \\
\hline & Yes & 48 & 91 & 139 & \\
\hline \multirow{2}{*}{ Death of close relative } & Negative & 126 & 74 & 200 & \multirow{2}{*}{0.000} \\
\hline & Positive & 14 & 41 & 55 & \\
\hline \multirow{2}{*}{ Death of Spouse } & Negative & 130 & 105 & 235 & \multirow{2}{*}{0.646} \\
\hline & Positive & 10 & 10 & 20 & \\
\hline \multirow{2}{*}{ Money Loss } & Negative & 124 & 79 & 203 & \multirow{2}{*}{0.000} \\
\hline & Positive & 16 & 36 & 52 & \\
\hline \multirow{2}{*}{ Job Loss } & Negative & 138 & 115 & 253 & \multirow{2}{*}{0.198} \\
\hline & Positive & 2 & 0 & 2 & \\
\hline \multirow{2}{*}{ Home Change } & Negative & 138 & 107 & 245 & \multirow{2}{*}{0.024} \\
\hline & Positive & 2 & 8 & 10 & \\
\hline \multirow{2}{*}{ Serious illness or accident } & Negative & 124 & 83 & 207 & \multirow{2}{*}{0.001} \\
\hline & Positive & 16 & 32 & 48 & \\
\hline \multicolumn{2}{|c|}{ Total } & 140 & 115 & 255 & \\
\hline
\end{tabular}

Table 17: Major life events among depressed participants and the statistical significant. 
Citation: Al-Abbudi SJ, Lami FH, Wady ZA (2018) Prevalence and Assessment of Severity of Depression Among Ischemic Heart Disease Patients Attending Outpatient Cardiology Department Baghdad Teaching Hospital, Baghdad, Iraq. J Psychiatry 21: 438. doi:10.4172/23785756.1000438

Page 8 of 10

\begin{tabular}{|c|c|c|c|c|c|}
\hline \multirow{2}{*}{\multicolumn{2}{|c|}{ Treatment }} & \multicolumn{2}{|c|}{ Depression } & \multirow{3}{*}{$\begin{array}{c}\text { Total } \\
95\end{array}$} & \multirow{2}{*}{$P$ value } \\
\hline & & NO & YES & & \\
\hline \multirow{2}{*}{ B-Blocker } & No & 60 & 35 & & \multirow{2}{*}{0.041} \\
\hline & Yes & 80 & 80 & 160 & \\
\hline \multirow{2}{*}{ Lipid Lowering Agents } & No & 44 & 38 & 82 & \multirow{2}{*}{0.784} \\
\hline & Yes & 96 & 77 & 173 & \\
\hline \multirow{2}{*}{ Nitrate } & No & 14 & 3 & 17 & \multirow{2}{*}{0.019} \\
\hline & Yes & 126 & 112 & 238 & \\
\hline \multirow{2}{*}{ ACE inhibitor } & No & 76 & 63 & 139 & \multirow{2}{*}{0.937} \\
\hline & Yes & 64 & 52 & 116 & \\
\hline \multirow{2}{*}{ Angiotensin } & No & 132 & 104 & 236 & \multirow{2}{*}{0.244} \\
\hline & Yes & 8 & 11 & 19 & \\
\hline \multirow{2}{*}{ Antiplatelet } & No & 4 & 8 & 12 & \multirow{2}{*}{0.124} \\
\hline & Yes & 136 & 107 & 243 & \\
\hline \multirow{2}{*}{ Calcium Channel Blocker } & No & 114 & 97 & 211 & \multirow{2}{*}{0.539} \\
\hline & Yes & 26 & 18 & 44 & \\
\hline \multirow{2}{*}{ Diuretics } & No & 102 & 82 & 184 & \multirow{2}{*}{0.783} \\
\hline & Yes & 38 & 33 & 71 & \\
\hline \multirow{2}{*}{ Anticoagulant } & No & 132 & 115 & 247 & \multirow{2}{*}{0.009} \\
\hline & Yes & 8 & 0 & 8 & \\
\hline \multicolumn{2}{|l|}{ Total } & 140 & 115 & 255 & \\
\hline
\end{tabular}

Table 18: Medications taken by depressed participant and the degree of statistical significant with depression.

\begin{tabular}{|c|c|c|c|c|c|c|}
\hline & & \multicolumn{2}{|c|}{ Depression } & \multicolumn{2}{|c|}{ Total } & \multirow{2}{*}{$P$ value } \\
\hline & & NO & YES & No. & $\%$ & \\
\hline \multirow{2}{*}{ Residence } & Urban & 130 & 103 & 233 & $91.3 \%$ & \multirow{2}{*}{0.352} \\
\hline & Rural & 10 & 12 & 22 & $8.7 \%$ & \\
\hline Family Hx Of Depression & No & 140 & 115 & 255 & $100 \%$ & -- \\
\hline \multirow{2}{*}{ Heart Surgery } & No & 134 & 115 & 249 & $97.6 \%$ & \multirow{2}{*}{0.025} \\
\hline & Yes & 6 & 0 & 6 & $2.4 \%$ & \\
\hline \multicolumn{2}{|l|}{ Total } & 140 & 115 & 255 & $100 \%$ & \\
\hline
\end{tabular}

Table 19: The statistical significance of some variables with the diagnosis of depression.

founded the prevalence of depression $40 \%$.

The prevalence of depression among ischemic heart disease patients of the current study was lower than; Carney (65\%) [21], Pena FM et al. (67\%) [22], Polikandrioti M et al. (65.4\%) [23], Rosenthal MH (45\%) [24], Manica et al. (45\%) [25].

Many study founded wide range of depressive disorder among ischemic heart disease patients; Hwang $\mathrm{B}$ and Choi $\mathrm{H}$ founded the prevalence of depression ranged from $24 \%$ to $68 \%$ [26]. Huffman JC (15\%-20\%) [7] Rudisch and Nemeroff (17-27\%) [27], Thomas SA et al. (13\%-77.5\%) [28], Shastri PC (40-65\%) [29], Rao M (30\% to 40\%) [30], Blumental JA (14\%-47\%) [31].

Regarding the severity of depression, HAMD-17 was applied on the DSM-IV positive respondents ischemic heart disease patients showed $14.9 \%$ have mild depression, $20 \%$ have moderate depression, $7.06 \%$ severe depression and $3.14 \%$ very severe depression. The degree of severity of depression is highly significant correlation with the type of ischemic heart disease $(\mathrm{P}=0.003)$. Type of ischemia is highly statistical significant correlated with severity of depression $(\mathrm{P}=0.006)$. Faur A founded mild depression $17.4 \%$, moderate depression $52.4 \%$, severe depression 15.5, and very severe depression 14.5\% [1]. IMHS 2006/7 [9] showed life time prevalence of severe depression 3.50\%, moderate depression $2.83 \%$, and mild depression $1.15 \%$. Pena FM et al. [22], studied sample of 103 patient in Brazil founded that 69 (67\%) were depressed; 35(34\%) had mild depression, 22 (21.3\%) had moderate depression, and $12(11.6 \%)$ patients presented with severe depression. Polikandrioti $\mathrm{M}$ et al. [23] showed that $34.6 \%$ absence of depression,
$27.3 \%$ mild depression, $20.9 \%$ moderate depression and $17.2 \%$ severe depression

\section{Age}

Most of the sample (93.7\%) where aged above 40 years. mean age $58.945 \pm 11.02$ years. More than $63 \%$ of depressed patients were of 51 70 years age group. The age group is highly significant correlation with the degree of depression $(\mathrm{P}=0.003)$. Since this period of age was at high risk for ischemic heart disease than other extremes, they have enough time for regular consultations, and they have high social supports from their families helping them for regular follow up of their health status. Pena FM et al. [22] founded the mean age was 65.4 years. Najeb GT [15] the mean age was $53.9 \pm 791$. Polikandrioti $\mathrm{M}$ et al. [23] founded 23.0\% were between 50-59 years. Rothenbacher D et al. [32] founded that the largest proportion of subjects with increased symptom scores were found in the age categories $40-49$ and $50-59$ years $(\mathrm{P}=0.0003$ and $\mathrm{P}=0.01$, respectively).

\section{Gender}

Male was about $70 \%$ of participants while female was about $30 \%$. Male depressed participants were $53 \%$ and female were $47 \%$. The gender is highly statistical significant correlation with the degree of depression ( $\mathrm{P}=0.014)$. Fraz K et al. founded $19.7 \% \%$ males and $7.5 \%$ females [5] Polikandrioti $\mathrm{M}$ et al. [23] founded $79.1 \%$ were male and only $20.9 \%$ patients were female. Najeb GT [15] founded male $62.1 \%$ and female $37.9 \%$ of depressed participants. Pena FM et al. [22] 36.9\% were male and $63.1 \%$ were female. Vural et al. [33] founded that female patients had higher scores of depression. 
Citation: Al-Abbudi SJ, Lami FH, Wady ZA (2018) Prevalence and Assessment of Severity of Depression Among Ischemic Heart Disease Patients Attending Outpatient Cardiology Department Baghdad Teaching Hospital, Baghdad, Iraq. J Psychiatry 21: 438. doi:10.4172/23785756.1000438

Page 9 of 10

\section{Marital status}

About $80 \%$ of sample and more than $75 \%$ of depressed were married. Marital status is significant statistical correlation with the diagnosis of depression $(\mathrm{P}=0.05)$. Najeb GT [15] founded that $69 \%$ were married than $31 \%$ were single. Polikandrioti $\mathrm{M}$ et al. [23] founded that $72.2 \%$ were married. Pena FM et al. [22] founded $49.5 \%$ were married. Dowlati $Y$ et al. [34], founded that there was significant statistical correlation of married status (married) with depression.

\section{Occupation}

House wife, the occupation that never be retired unless be disabled, form $41.7 \%$, retired $24.3 \%$, private work $21.7 \%$ among depressed ischemic heart disease patients. The occupation is highly statistical significant correlation with the diagnosis of depression $(\mathrm{P}=0.001)$ and the degree of severity of depression $(\mathrm{P}=0.000)$. Najeb GT [15] founded $53.4 \%$ not employed, $20.7 \%$ private work and $12 \%$ retired. Polikandrioti $\mathrm{M}$ et al. [23] founded higher levels of depression were observed for those retired $(\mathrm{p}=0.028)$.

\section{Education}

About $75 \%$ of the sample and about $85 \%$ of depressed ischemic heart disease patients were of low educational level or illiterate. The level of education of ischemic heart disease patients is of statistically significant correlation with the diagnosis of depression $(\mathrm{P}=0.000)$. Polikandrioti $\mathrm{M}$ et al. [23] founded that $47.5 \%$ of participants had received only basic education, $25.2 \%$ had received high school education, $27.3 \%$ had attended some college or university. Pena FM et al. [22] only $26.2 \%$ were illiterate. Najeb GT [15] founded $46 \%$ illiterate, about $70 \%$ of low education.

\section{Income}

All participants and depressed ischemic heart disease patient $(100 \%)$ were have monthly income $=533.301 \pm 56.951$ Iraqi dinar. The income is highly statistical significant correlation with depression among ischemic heart disease patients $(\mathrm{P}=0.000)$. Pena FM et al. [22] founded $43.7 \%$ have monthly income. Duration of ischemic heart disease: Current study founded Depression with ischemic heart disease of duration mean of 5 years ( $53.38 \pm 68.746$ months), range from one month to 30 years. The duration of ischemic heart disease is statistically significant correlated with depression $(\mathrm{P}=0.001)$.

\section{Comorbidity}

$86.1 \%$ have comorbidity. Statistically significant comorbidity with depression were; hypertension $(61.7 \%)(\mathrm{P}=0.000)$, diabetes mellitus (43.4\%) $(\mathrm{P}=0.003)$, and heart failure (13.9\%) $(\mathrm{P}=0.026)$. Pena FM et al. [23] founded $92.2 \%$ hypertension and $34 \%$ diabetes mellitus.

\section{Life events}

About $80 \%$ of depressed ischemic heart disease patients have at least one major life event. The life events were statistically significant correlation with depression $(\mathrm{P}=0.000)$. Significant life events were; death of close relative $(35.6 \%)(\mathrm{P}=0.000)$, loss of money $(31.3 \%)$ $(\mathrm{P}=0.000)$, changing home or displaced $(6.9 \%)(\mathrm{P}=0.024)$, and serious illness or accidents (27.8\%) $(\mathrm{P}=0.001)$. Michael AJ et al. [35] founded that $32 \%$ of psychiatric illness attributed to stressful life events. Michael AJ et al. [36]. Founded that $25-26 \%$ of the patients had life events.

\section{Medications}

Depressed ischemic heart disease patients were of medication for the ischemia. Statistically significant correlation with depression was; B-blocker $(\mathrm{P}=0.041)$, nitrate $(\mathrm{P}=0.019)$, and anticoagulant $(\mathrm{P}=0.009)$. Dowlati et al. [34] founded no significant statistical correlation of medications (B-blockers and ACE inhibitors) with depression. Vural et al. [33]. Founded that Statin usage (47.5\% of all patients) was not associated with increased depression scores.

\section{Smoking}

About $32.1 \%$ of depressed ischemic heart disease patients were smokers. Number of cigarettes per day was $11.435 \pm 19.754(\mathrm{P}=0.05)$ Pena FM et al. [22] founded 35\% were smokers.

\section{Alcohol}

Only $1.7 \%$ of depressed ischemic heart disease patients were alcoholics on regular pattern. Alcohol consumption was statistically not significant $(\mathrm{P}=0.246)$. Pena FM et al. [22] founded $21.4 \%$ were alcoholics.

\section{Residence}

More than $90 \%$ were live in the urban than rural areas. These finding were statistically investigated show no significant correlation with depression $(\mathrm{P}=0.352)$. Polikandrioti $\mathrm{M}$ et al. [23] founded; urban $34.5 \%$ and rural 23.7. Herva A [36] founded the prevalence of major depressive disorder was $4.7 \%$ among urban subjects, and $4.1 \%$ among rural subjects.

\section{Family history}

All participants (100\%) were of negative family history of mental illness. No statistical value obtained.

\section{Heart surgery}

Six $(2.3 \%)$ underwent surgical procedures. No one of them was depressed according to DSM-IV criteria for depression. This finding is statistically significant $(\mathrm{P}=0.025)$.

\section{Conclusion}

Current study shows high prevalence of depression (45.1\%) among out-patient ischemic heart disease patients. Depressed ischemic heart disease patients were of statistically significant correlation with age, sex, marital status, occupation, education, income, and duration of ischemia, comorbidity with other illnesses, cardiac surgery, and stressful life events.

With lack of mental health services, this issue is more important for the general practitioners and cardiologists to understand the importance of risks of untreated depression in ischemic heart disease patients. Complicating this picture is the prevailing social stigma associated with mental illness in Iraq.

Review of studies found that the association between current heart disease and prevalence of major depression was fairly consistent across many countries and cultures in Europe, the Americas, the Middle East, Africa, and Asia.

\section{Recommendations}

In the face of high prevalence of depression among ischemic heart disease patients, educate the public and raising awareness of the healthcare providers is recommended.

Important future research directions including the need of Large-scale, prospective studies in patients with ischemic heart 
Citation: Al-Abbudi SJ, Lami FH, Wady ZA (2018) Prevalence and Assessment of Severity of Depression Among Ischemic Heart Disease Patients Attending Outpatient Cardiology Department Baghdad Teaching Hospital, Baghdad, Iraq. J Psychiatry 21: 438. doi:10.4172/23785756.1000438

Page 10 of 10

disease to improve understanding of the reciprocal effects between depression, ischemic heart disease, and symptom burden, functional impairment, and self-care regimens. Large, randomized, controlled studies are needed in representative populations with ischemic heart disease and major depression to test the effect of enhancing quality of depression care and improving outcomes of depression on symptom burden, function, self-care activities, direct and indirect costs, medical complications, and mortality. Research is needed to determine which treatment is most effective and whether the treatment of depression will improve morbidity and mortality in patients with ischemic heart disease and depression.

\section{References}

1. Faur A (2016) Correlation between depression and stress test at patients with ischemic heart. Journal of the Romanian Sports Medicine Society (Medicina Sportiva) 11: 2715-2720.

2. Bozzini S, Falcone C (2015) The interface of coronary artery disease and depression: Pathophysiology and diagnosis. J Cardiovasc Disord 2: 1015.

3. Colquhoun DM, Bunker SJ, Clarke DM, Glozier N, Hare DL, et al. (2013) Screening, referral and treatment for depression in patients with coronary heart disease. Med J Aust 198: 483-484.

4. Hare DL, Toukhsati SR, Johansson P, Jaarsma $T$ (2014) Depression and cardiovascular disease: A clinical review. Eur Heart J 21: 1365-1372.

5. Fraz K, Khan SA, Sikander S (2013) Screening for depression in coronary artery disease patients using PHQ-9. LIP Health 4: 3-6.

6. O'Neil A (2013) The relationship between coronary heart disease (CHD) and major depressive disorder (MDD): Key mechanisms and the role of quality of life. Eur J Psychol 9: 163-184.

7. Huffman JC, Celano CM, Beach SR, Motiwala SR, Januzzi JL (2013) Depression and cardiac disease: epidemiology, mechanisms, and diagnosis. Cardiovasc Psychiatry Neurol 2013: 695925.

8. Raj HSS, Sajimon PP (2010) Anxiety and depression in ischeamic hear disease. J Indian Acad Appl Psychol 36: 239-244.

9. Ministry of Health (2008) Iraq mental health survey 2006/7 report.

10. No authors mentioned (1994) Diagnostic and statistical manual of menta disorders (4th edn.). American Psychiatric Association, Washington, DC.

11. Hamilton M (1960) A rating scale for depression. J Neurol Neurosurg Psychiatry 23: $56-62$

12. Salman TS (2009) Prevalence of mental illnesses among adults attending primary health care centers in Baghdad.

13. No authors listed (2008) Violence related mortality in Iraq from 2002 to 2006 Iraq Family Health Survey Study Group. N Eng J Med 358: 484-493.

14. Liang JJ, Tweet MS, Hayes SE, Gulati R, Hayes SN (2014) Prevalence and predictors of depression and anxiety among survivors of myocardial infarction due to spontaneous coronary artery dissection. J Cardiopulm Rehabil Prev 34 138-142.

15. Najeeb GT (2006) Depression in acute myocardial infarction: a case series study.

16. Lawson R, Musselman D, Otte C, Bruce E, Ali S, et al. (2009) Depression and whole blood serotonin in patients with coronary heart disease from the heart and soul study. Psychosom Med 3: 260-265.
17. Watkins LL, Schneiderman N, Blumenthal JA, Sheps DS, Catellier D, et al. (2003) Cognitive and somatic symptoms of depression are associated with medical co-morbidity in patients after myocardial infarction. Am Heart J 146: $48-54$

18. Leegte IL, Jaarsma T, Sanderman R, Linssen G, Veldhuisen DJ (2006) Depressive symptoms are prominent among elderly hospitalized heart failure patients. Eur J Heart Fail 8: 634-640.

19. Whooley MA (2006) Depression and cardiovascular disease: Healing the broken-hearted. JAMA 295: 2874-2881.

20. Kemp DE, Malhotra S, Franco KN, Tesar G, Bronson DL (2003) Heart disease and depression: Don't ignore the relationship. Cleve Clin J Med 70: 745-750.

21. Carney RM, Freedland KE, Sheline YI, Weiss ES (1997) Depression and coronary heart disease; A review for cardiologists. Clinical Cardiol 20: 196-200.

22. Pena FM, Modenesi Rde F, Piraciaba MC, Marins RM, Souza LB, et al. (2010) Prevalence and variables predictive of depressive symptoms inpatients hospitalized for heart failure. Cardiol J 17: 18-25.

23. Polikandrioti M, Christou A, Morou Z, Kotronoulas G, Evagelou H, et al. (2010) Evaluation of depression in patients with heart failure. Health Science Journal 4: 37-47.

24. Rosenthal MH (2003) The challenges of comorbid disorders in patients with depression. J Am Osteopath Assoc 103: 10-15.

25. Manica ALL, Carolina GSL, Frey BN, Juruena MN (1999) The role of depression in coronary artery disease. Arq Bras Cardiol 73: 244-245.

26. Hwang B, Choi H (2016) Depressive symptoms among patients with heart failure in Korea: An integrative review. Healthcare 52: 2-9.

27. Rudisch B, Nemeroff CB (2003) Epidemiology of comorbid coronary artery disease and depression. Biol Psychiatry 54: 227-240.

28. Thomas SA, Chapa DW, Friedmann E, Durden C, Ross A, et al. (2008) Depression in patients with heart failure: Prevalence, pathophysiological mechanisms, and treatment. Crit Care Nurse 28: 40-55.

29. Shastri PC (2000) Depression and cardiac disorders. Am Heart J 140: 563-572.

30. Rao M (2008) Depression in the physically III. Primary Psychiatry 15: 44-50.

31. Blumental JA (2008) Depression and coronary heart disease: Association and implications for treatment. Cleve Clin J Med 75: 48-52.

32. Rothenbacher D, Hahmann H, Wüsten B, Koenig W, Brenner H (2007) Symptoms of anxiety and depression in patients with stable coronary hear disease: prognostic value and consideration of pathogenetic links. Euro Cardiovasc Prev Rehabil 14: 549-550.

33. Vural (2008) Depression, anxiety, and panic agoraphobia. Anadolu Kardiyol Derg 8: 7-43.

34. Dowlati Y, Hermann N, Swardfager W, Thomson S, Oh IP, et al. (2010) Relationship between hair cortisol concentrations and depressive symptoms in patients with coronary artery disease. Neuropsychiatr Dis Treat 6: 393-400.

35. Michael AJ, Krishnaswamy S, Alyeope FB, Yusoff K, Muthuswamy TS, et al. (2004) Patients with acute myocardial infarction and unstable angina have significant levels of anteceding stress. Hong Kong J Psychiatry 14: 23-28.

36. Herva A (2007) Depression in association with birth weight, age, at menarche obesity and metabolic syndrome in young adults. The Northern Finland 1966 Birth Cohort Study, Acta Univrstatis Ouluensis D Medica. 